\title{
Secure pseudonymisation for privacy-preserving probabilistic record linkage
}

DOI:

10.1016/j.jisa.2017.01.002

\section{Document Version}

Accepted author manuscript

Link to publication record in Manchester Research Explorer

\section{Citation for published version (APA):}

Smith, D. (2017). Secure pseudonymisation for privacy-preserving probabilistic record linkage. Journal of Information Security and Applications. https://doi.org/10.1016/j.jisa.2017.01.002

\section{Published in:}

Journal of Information Security and Applications

\section{Citing this paper}

Please note that where the full-text provided on Manchester Research Explorer is the Author Accepted Manuscript or Proof version this may differ from the final Published version. If citing, it is advised that you check and use the publisher's definitive version.

\section{General rights}

Copyright and moral rights for the publications made accessible in the Research Explorer are retained by the authors and/or other copyright owners and it is a condition of accessing publications that users recognise and abide by the legal requirements associated with these rights.

\section{Takedown policy}

If you believe that this document breaches copyright please refer to the University of Manchester's Takedown Procedures [http://man.ac.uk/04Y6Bo] or contact uml.scholarlycommunications@manchester.ac.uk providing relevant details, so we can investigate your claim.

\section{OPEN ACCESS}




\title{
Secure Pseudonymisation for Privacy-Preserving Probabilistic Record Linkage
}

\author{
D. Smith ${ }^{1, *}$ \\ School of Social Sciences \\ Humanities Bridgeford Street \\ University of Manchester \\ Oxford Road \\ Manchester \\ M13 9PL, UK
}

\begin{abstract}
Record linkage is becoming an increasingly important tool in many areas of research - particularly medical research, where the relevant data often reside in more than one location. In the absence of a reliable and unique identifier probabilistic approaches to linkage are often employed. This linkage generally exploits the information contained in the fields that are common to a record pair. In classical record linkage the values in common fields are simply compared for equality. As values might contain typographical (or other) errors the performance of classical record linkage can often be significantly improved if similarities between value pairs are also exploited.

In applications where the data used for matching must be kept private the raw values are replaced by pseudonyms. For better linkage performance these pseudonyms should also convey information regarding similarities. Existing approaches are often based on Bloom filters, yet these are susceptible to attack. Secure schemes based on Bloom filters inevitably involve additional security measures. Here we introduce a new scheme that produces pseudonyms that are far more secure than Bloom filters. It can be used a drop-in replacement for many schemes that use Bloom filters. The new scheme allows similarity scores to be estimated from pairs of pseudonyms with negligible bias and with known variance for a given similarity score.
\end{abstract}

\section{(C)}

Keywords:

Record linkage, privacy, Bloom filter, hash function

\section{Introduction}

Record linkage is the task of identifying distinct records that correspond to the same entity. The records might be contained in a single dataset, in which case the process might be termed de-duplication. More usually records are matched across distinct datasets $\mathcal{A}$ and $\mathcal{B}$. Each pair of records $(a, b)$ in the Cartesian product of $\mathcal{A}$ and $\mathcal{B}$ is allocated

\footnotetext{
*Principal corresponding author

Email address: duncan.g. smith@manchester.ac.uk (D. Smith)

${ }^{1}$ The research leading to these results has received funding from the European Union's Seventh Framework Programme (FP7/2007-2013) under grant agreement no. 262608 (DwB - Data without Boundaries)
} 
to either a set of correct matches, $\mathcal{M}$, or a set of incorrect matches, $\mathcal{U}$. Classification is generally performed on the basis of comparisons on the values of the fields common to $\mathcal{A}$ and $\mathcal{B}$. In classical (Fellegi-Sunter) record linkage [1] comparisons are based on equality and it is assumed that the result of a comparison on a given field is independent of the comparisons on other fields given the match status. This leads to the following expression for the posterior odds of a correct match,

$$
\begin{array}{r}
\frac{\operatorname{Pr}((a, b) \in \mathcal{M} \mid a, b)}{\operatorname{Pr}((a, b) \in \mathcal{U} \mid a, b)}=\left(\prod_{i=1}^{n} \frac{\operatorname{Pr}\left(a_{i}, b_{i} \mid(a, b) \in \mathcal{M}\right)}{\operatorname{Pr}\left(a_{i}, b_{i} \mid(a, b) \in \mathcal{U}\right)}\right) \\
\times \frac{\operatorname{Pr}((a, b) \in \mathcal{M})}{\operatorname{Pr}((a, b) \in \mathcal{U})}
\end{array}
$$

where the records are aligned so that the common variables are indexed $i=(1, \ldots, n)$. All the necessary parameters can be estimated from the data being linked using an expectation maximisation (EM) approach [2]. The input into the EM algorithm is a binary (comparison) value for each record pair for each comparison field. Record pairs are allocated to either $\mathcal{M}$ or $\mathcal{U}$ (and sometimes a third category of uncertain matches) on the basis of thresholds on the posterior probability of a correct match. Equivalently the thresholds might be placed on the posterior odds or the match weight ( $\log$ of the likelihood ratio). Record pairs that are classified as uncertain matches are subjected to clerical review - a human inspects the record pair and manually allocates it to either $\mathcal{M}$ or $\mathcal{U}$.

Values will often contain typographical (or other) errors, so record linkage can be improved by exploiting measures of similarity. A simple approach is to treat all pairs of values with similarity scores above a given threshold as equal. In this case the comparisons are still binary and the standard EM record linkage machinery can be exploited. Alternative approaches have exploited similarities in various ways, such as piecewise interpolation on match weights [3], improving EM parameter estimation via selective sampling of record pairs [4], and extending EM estimation to multinomial comparisons [5]. Non-classical approaches tend to use similarity scores more directly (e.g. [6]). Approaches that exploit similarities are not generally bound to specific types of score, but do often require that scores must be in the interval [0,1]. Measures based on string edit distances such as Jaro [7] and Jaro-Winkler [3] have been found to be effective.

In privacy-preserving record linkage $\mathcal{A}$ and $\mathcal{B}$ are held by distinct organisations (Alice and Bob) which are subject to restrictions on data sharing. Typically the common fields will contain identifying information such as name and (components of) address. These are required for linkage but cannot be shared due to privacy concerns. If linkage can be achieved in a privacy-preserving manner, then the identifying information can be removed from the linked records before they are released to end users. The usual approach to privacy-preserving record linkage is to replace the values in the identifying fields with pseudonyms that can be used for linkage. The pseudonymised data are then passed to a third party, Carol, who performs the actual linkage. For effective linkage the pseudonyms must allow the computation (or estimation) of similarity scores. This paper introduces a pseudonymisation scheme that allows the estimation of Jaccard (Tanimoto) scores from pairs of pseudonyms. The scheme is compared to the Bloom filter approach that has become an almost de facto standard for privacy-preserving record linkage.

\section{Background}

The simplest approach to string pseudonymisation is to use a deterministic hash function such as MD5. The problem is that this is susceptible to a dictionary attack - the string values can be recovered by hashing candidate strings and matching the hashes against the pseudonyms. An alternative is to use a deterministic hash function, such as HMAC, that depends on a secret key that can be securely shared between Alice and Bob. Dictionary attacks become infeasible, but pairs of pseudonyms still convey no similarity information other than equality / inequality.

Churches and Christensen [8] describe a scheme whereby Alice and Bob can pseudonymise strings so that Carol can generate similarity scores. Alice and Bob use a common hash function that is dependent on a secretly shared key. Each string is tokenised into a bigram set $S$ - the set containing all adjacent pairs of characters - before hashing each non-empty member of the powerset of $S$. A pseudonym consists of a list of 2-tuples, each containing a hash and the size of the corresponding subset of tokens. For example, the string 'john' would produce the token set \{'jo','oh','hn' \} and Carol would be provided with the hashes and sizes of the ordered tuples ('jo'), ('oh'), ('hn'), ('jo','oh'), ('jo','hn'), 
('oh','hn') and ('jo','oh','hn'). For a pair of pseudonyms a common subset of tokens appears as a common hash value. The largest size associated with a common hash value gives the size of the intersection of token sets. The largest size associated with a pseudonym gives the size of the token set. Thus Carol can calculate the Dice coefficient,

$$
D_{A, B}=\frac{2|A \cap B|}{|A|+|B|} .
$$

There are weaknesses in this basic approach, which are discussed in Churches and Christensen [8]. One issue is that Carol is explicitly provided with the sizes of the token sets. Depending on what she can reasonably assume about the tokenisation scheme, Carol could make inferences about the lengths of the underlying strings and thus the value of the corresponding variable. She could also launch frequency attacks against the tokens. Common tokens will lead to common hashes for token subsets of size 1, commonly co-occurring pairs of tokens will lead to common hashes for token subsets of size 2, and so on. Relevant prior information might be gleaned from sources such as telephone directories or, perhaps, via the application of Benford's law for appropriate numerical fields ${ }^{2}$.

Schnell et al. [9] propose the use of Bloom filters to pseudonymise strings. A Bloom filter is a probabilistic set data structure that can represent a set as a bitstring much more compactly than if a bit position were reserved for each possible feature. The number of bits, $m$, is fixed, and $k$ hash functions are used to map each token to $k$ indices in the range $[0, m-1]$. When a token is added to a Bloom filter each of the corresponding $k$ bit positions is set to 1 (they are all initially 0). Schnell et al. [9] estimate the Dice coefficient for a pair of Bloom filters (bitstrings) $A$ and $B$ as twice the number of set bits in the bitwise AND of $A$ and $B$ divided by the sum of the numbers of set bits in $A$ and $B$.

Another commonly used set similarity score is the Jaccard score,

$$
J_{A, B}=\frac{|A \cap B|}{|A \cup B|} .
$$

The usual approach for estimating Jaccard scores from Bloom filters is to take the number of set bits in the bitwise AND of $A$ and $B$, then divide the result by the number of set bits in the bitwise OR of $A$ and $B$. Swamidass and Baldi [10] present a 'corrected' estimator that is far less biased than the usual estimator. Furthermore, they show that in their application area (chemical retrieval) the corrected estimator generates more meaningful results. It appears that in record linkage the corrected estimator is not generally used, and no comparison of the performance of the two estimators has been carried out.

Bloom filters suffer from security issues. It is possible to estimate the size of a token set from $m, k$ and the number of set bits. Although a dictionary attack can be easily avoided by using suitable hash functions and a shared secret key, common tokens result in commonly set $k$-subsets of bits and frequency attacks against tokens can be launched. Bloom filters can also suffer from the issue of filling with 1s. If token sets are large in relation to $m$ the proportion of set bits becomes large and similarity score estimates have high variance. In practice $m$ and $k$ need to be chosen carefully. In any case the variance of estimates will itself be variable.

Both Churches and Christensen [8] and Schnell et al. [9] propose methods to mitigate against the re-identification of variables and frequency attacks on tokens. However, these inevitably perturb the data sent to Carol, which can negatively impact on the estimation of similarity scores.

The following sections describe an alternative pseudonymisation approach. Strings are tokenised, and pseudonyms are generated from the token sets. The lengths of token sets cannot be estimated from the pseudonyms and the risk from frequency attacks on tokens is dramatically reduced. A pseudonym is (for all practical intents and purposes) a uniform random integer. Thus the expected number of set bits is half the length of the pseudonym. This avoids the problem of large token sets filling the pseudonym with 1s. Jaccard scores can be estimated from a pair of pseudonyms with negligible bias. The variance of estimates depends only on the underlying Jaccard score and the length of the pseudonyms.

\footnotetext{
${ }^{2}$ Adequately capturing similarity for numerical fields might require an alternative tokenisation scheme
} 
The one form of attack for which risk is not reduced is a frequency attack on the underlying strings. For instance, it might be possible to re-identify the variable 'gender' due to it containing only two distinct values. Given background knowledge of the data source it might be possible to identify which values correspond to males / females. Reducing this risk via any pseudonymisation scheme requires either allowing distinct strings to generate equal pseudonyms (with non-negligible probability), or allowing equal strings to generate distinct pseudonyms (with non-negligible probability). An alternative means of reducing this risk is the addition of dummy records to disguise the underlying frequencies (e.g. [8]). Although this always remains a choice, it can negatively impact record linkage in terms of both computational cost and the estimation of the Fellegi-Sunter parameters. The main point here is that we cannot expect a pseudonymisation scheme to deal with this source of risk. So in this paper we only consider the risks that can be addressed through the choice of pseudonymisation scheme - the re-identification of variables and frequency attacks on tokens.

\section{Secure pseudonymisation}

This section describes the relevant background theory before going on to describe the secure pseudonymisation scheme in detail. In Section 3.1 we describe minwise hashing [11] and the estimation of the underlying Jaccard score for a pair of tokenised and minwise hashed strings. In Section 3.2 we describe an implementation of minwise hashing suggested by Pătraşcu and Thorup [12]. In Section 3.3 we demonstrate that minwise hashing allows the estimation of token set sizes and is susceptible to frequency attacks on tokens. In Section 3.4 the $b$-bit minwise hashing scheme of Li and König [13] is described for the specific case of $b=1$. Assumptions are justified that lead to an alternative mathematical treatment to that presented in Li and König's paper. This also allows us to demonstrate that 1-bit minwise hashing generates pseudonyms that do not permit the estimation of token set sizes and that dramatically reduce the risk of frequency attacks on tokens. This is the main contribution of this paper.

\subsection{Locality sensitive hashing}

Locality sensitive hash functions generate hashes so that similar inputs will hash to the same value with nonnegligible probability. Minwise hashing [11] is a locality sensitive hashing method that generates a random permutation of a set of elements and returns the hash of the first ordered element. Thus pairs of sets with non-empty intersection will generate equal hashes with non-negligible probability. In order to generate a consistent permutation across a number of sets the number of possible elements must be finite. This universe of elements can be permuted, and distinct sets of elements reordered according to the permutation of the universe. If we consider the union of two sets $A$ and $B$, then the probability that any given element in the union is ordered first is simply $1 /|A \cup B|$. We have $|A \cap B|$ elements that will generate equal minwise hashes if ordered first. Thus the probability of a minwise hash collision is equal to the Jaccard score for $A$ and $B$.

A practical difficulty with the permutation generation can be the size of the universe. To allow for large universes hashing is often used to generate the permutation. If we use a suitable hash function to hash the elements and then reorder by hash value, then we have generated a random permutation of the elements. Ordering will be consistent across distinct sets of elements. Of course, under this scheme the hash of the first ordered element is simply the smallest value in the set containing the hashes of the tokens. So no explicit permutation of elements is actually performed.

In order to be able to estimate the Jaccard score from minwise hashes, many of them must be generated using the members of a suitable family of hash functions. Thus a pseudonym is a list of minwise hash values, and Jaccard score estimation is based on the number of hash collisions. Assume that the underlying hash functions (used to hash the set elements) are chosen so that the probability of unintentional collisions is negligible. Then, if we generate $m$ hash values using $m$ suitable minwise hash functions, the number of hash collisions $X$ is distributed,

$$
X \sim \operatorname{Bin}\left(m, J_{A, B}\right)
$$

As the number of collisions follows a Binomial distribution we can define $m$ to be an integer large enough to produce an estimate of $J_{A, B}$ to a given level of precision. The estimator and its variance are simply, 


$$
\widehat{J}_{A, B}=\frac{X}{m}
$$

and,

$$
\operatorname{Var}\left(\widehat{J}_{A, B}\right)=\frac{J_{A, B}\left(1-J_{A, B}\right)}{m} .
$$

\subsection{Minwise hash implementation}

Wegman and Carter [14] define a hash function as being $k$-independent if for any distinct $x_{1}, \ldots, x_{k}$ keys the values $h\left(x_{1}\right), \ldots, h\left(x_{k}\right)$ are independent random variables uniformly distributed over the relevant range. But this is not the form of independence we require for minwise hashing.

Consider a set $S$ of keys and $y \notin S$. Then minwise independence holds if,

$$
P\left(h(y)<\min _{x \in S}\{h(x)\}\right)=\frac{1}{|S|+1}
$$

where $\min _{x \in S}\{h(x)\}$ is the minwise hash for $S$.

Pătraşcu and Thorup [12] show that simple tabulation hashing is suitable for minwise hashing despite being only 3-independent. Simple tabulation hashing works by splitting a fixed length binary key into $c$ sub-keys. Each sub-key is used as an index into one of $c$ lookup tables of random integers. The retrieved integers are XORed to produce a hash for the key.

For example the 32-bit binary key,

$$
10001010101010110111010101010011
$$

can be split into four 8-bit sub-keys.

$$
\text { 10001010, 10101011, 01110101, } 01010011
$$

Each sub-key is used to look up a random integer in a corresponding lookup table containing $2^{8}$ randomly generated integers. Thus we need $4 \times 2^{8}$ random $q$-bit integers to generate a $q$-bit hash. In order to generate identical simple tabulation hash functions Alice and Bob simply need to use the same PRNG and a securely shared seed. The minwise independence assumption is more reasonable for small $c$ (see [12]), although this requires the generation of larger numbers of random integers.

As we might be hashing variable length tokens we hash them to an intermediate form that is a suitable key for tabulation hashing. The current implementation uses the 64 least significant bits of an MD5 hash. Eight lookup tables are used, each containing 256 64-bit random integers. Generating $m$ hash functions simply requires the generation of $m$ sets of lookup tables. A more recent variation of simple tabulation hashing, named twisted tabulation hashing, is reported to be even better for minwise hashing [15], although it will not be described because it is not used in our current implementation.

Table 1 shows simple tabulation minwise hashes for the bigram-tokenised strings 'john' and 'jon'. The common bigram 'jo' results in one hash collision. (Note: 32-bit hashes are used in Table 1 for clarity of exposition.) The true Jaccard score is $1 / 4$. The Jaccard score estimate based on the first 5 hash functions is $1 / 5$. Of course the variance for this estimate is large given only 5 hash functions. 
Table 1. Minwise hashes for token sets $\mathrm{S} 1=\{$ 'jo','oh','hn' $\}$ and S2 $=\{$ 'jo','on' $\}$

\begin{tabular}{|c|c|c|c|c|c|c|}
\hline & $\mathrm{H}_{1}$ & $\mathrm{H}_{2}$ & $\mathrm{H}_{3}$ & $\mathrm{H}_{4}$ & $\mathrm{H}_{5}$ & $\ldots$ \\
\hline$S_{1}$ & 2395193425 & 2091676248 & 458611983 & 2378308598 & 959567390 & \\
\hline $\mathrm{S}_{2}$ & 3230094103 & 4023613335 & 2310837953 & 2378308598 & 763155152 & \\
\hline$S_{N}$ & & & & & & \\
\hline
\end{tabular}

\subsection{Security issues}

If we hash the members of a token set of size $n$ using a randomly generated $q$-bit simple tabulation hash function, then it is reasonable to treat the $n$ hash values as independent and uniformly distributed integers in the range $\left[0,2^{q}-1\right]$. Then each published value (minwise hash) is the 1st order statistic of the token set hashes, which is distributed,

$$
P\left(X_{(1)}=x\right)=\left(1-\frac{x}{2^{q}}\right)^{n}-\left(1-\frac{x+1}{2^{q}}\right)^{n} .
$$

The Binomial theorem can be used to generate the approximation,

$$
P\left(X_{(1)}=x\right) \approx \frac{n}{2^{q}}\left(1-\frac{x}{2^{q}}\right)^{n-1}
$$

This is a very close approximation for reasonable values of $q$ (i.e. where $2^{-2 q}<<2^{-q}$ ), and can be derived directly if we assume no unintended collisions on the minimum hash value.

If we have a list of such hashes indexed from 1 to $m$, then we have the following log likelihood function,

$$
\ln (f(x \mid n))=m \ln (n)-m q \ln (2)+(n-1) \sum_{i=1}^{m} \ln \left(1-\frac{x_{i}}{2^{q}}\right) .
$$

A little calculus produces the estimator,

$$
\widehat{n}=\frac{-m}{\sum_{i=1}^{m} \ln \left(1-\frac{x_{i}}{2^{q}}\right)}
$$

where the true maximum likelihood estimate for $n$ is either $\lfloor\widehat{n}\rfloor$ or $\lceil\widehat{n}\rceil$, which can be decided by evaluating the log likelihood function at $\lfloor\widehat{n}\rfloor$ and $\lceil\widehat{n}$.

Compare this with the estimator for the number of items in a Bloom filter [10],

$$
\widehat{n}=\frac{m \ln \left(1-\frac{N}{m}\right)}{-k}
$$

where $m$ is the length of the filter, $k$ is the number of hash functions, and $N$ is the number of set bits.

As an experiment a sample of 300 names was tokenised and hashed using minwise hashing. Names were sampled from the list of Titanic passengers ${ }^{3}$ (after removing names with non-ASCII characters). Names were then padded with 
a leading and trailing underscore before tokenising into bigrams (padding ensures that all characters appear in exactly two bigrams). Estimated sizes (unrounded) are plotted against actual token set sizes in Figure 1.

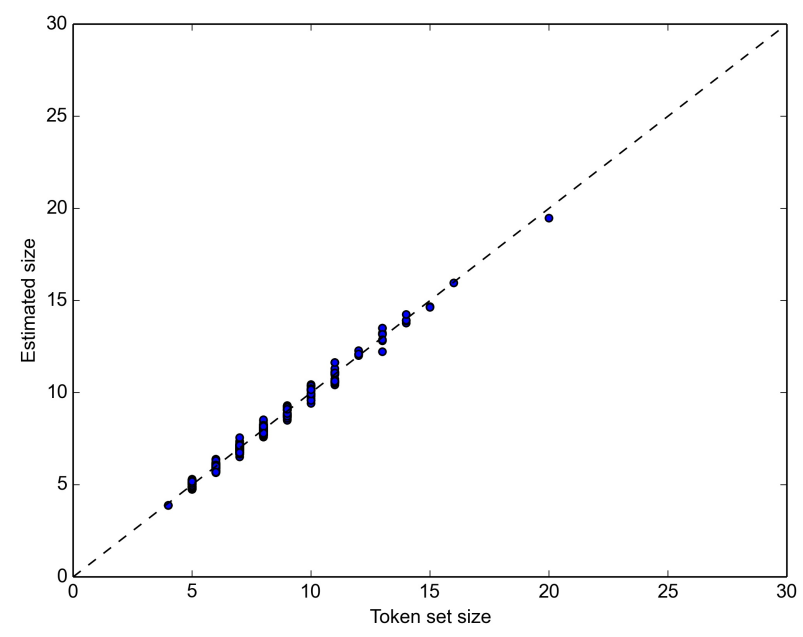

Figure 1. Estimated sizes (unrounded $n$ ) of token sets against actual sizes

Carol could clearly use this estimator to help identify the relevant variable. It is clearly not gender or a variable containing short numeric strings that might denote age or some component of date of birth. Unusually short or long token sets might provide clues as to the underlying values - for instance individuals over 100 if the variable had been identified as age. Of course, the numbers of distinct rows (lists of minwise hashes) will clearly provide a clue as to the relevant variable. This risk cannot be dealt with via the pseudonymisation scheme.

Another ploy would be to attack the columns of Table 1 - the individual hash functions. For any given column, frequently occurring tokens will generate frequently occurring hashes. Minwise hashing dictates that only the hash of a single token is returned as the hash value for a token set. So hash frequencies do not necessarily reflect underlying token frequencies. However, the frequency of the minimum hash value in a column corresponds exactly to the frequency of the corresponding token.

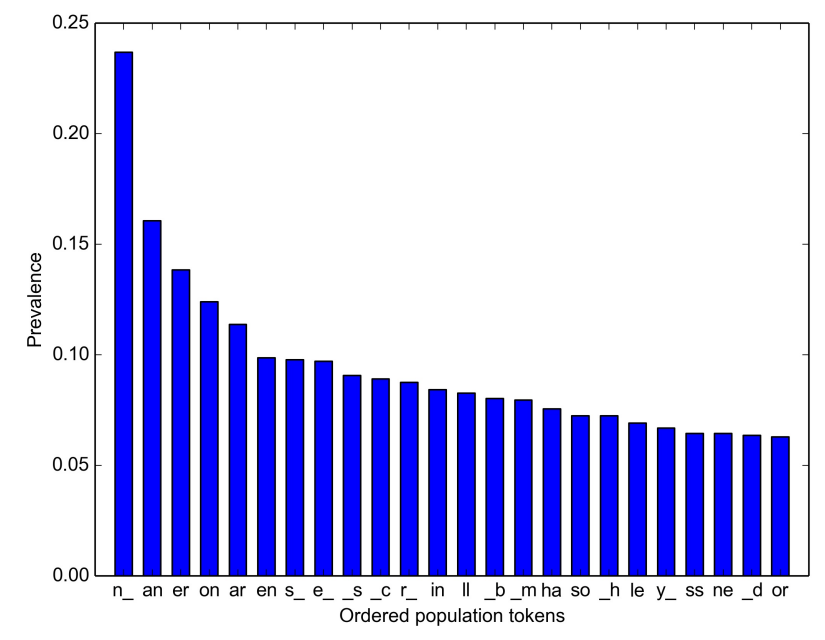

Figure 2. Proportion of occurrences of the 25 most common tokens in population data, ordered from most frequent to least frequent 
Figure 2 shows the proportion of occurrences of the most frequent tokens in the list of all 1258 Titanic passenger surnames (containing all ASCII characters). The most common is 'n_' at 23.7\%. This is due to the high proportion of surnames with endings such as 'ton' or 'son'. Carol might possess such prior information derived from sources such as telephone directory entries (assuming she has already identified the variable).

If we plot the proportions of occurrences of the smallest valued hashes against column index for our sample of 300 names we get Figure 3. Confidence intervals for the underlying proportions can be generated and compared with population frequencies. It is apparent that we have 3 columns for which the smallest hash value corresponds to 'n_'. Of course, Carol does not need to stop here. The frequencies of the second smallest hash values can also be treated as Binomial random variates and confidence intervals generated. The observed frequency of the second smallest hash value in a column is the frequency of occurrence in token sets that do not contain the token corresponding to the smallest valued hash, and so on.

There is quite a lot of information that can be exploited by Carol. The above are just basic forms of attack used to illustrate weaknesses. It is not clear that the minwise hashing scheme as presented above is any more secure than using Bloom filters. However, the means by which the estimated scores are generated hints at simple adjustments that will reduce risk. To estimate the Jaccard score we simply need to know which hash values collide. The values of the hashes are immaterial, and only make the scheme vulnerable to variable re-identification and frequency attacks on tokens. A consistently applied permutation of the hash digits would disguise which values in a column were lowest and would do something to reduce risks. Removal of the most significant bit would have a similar impact - whilst slightly increasing the risk of unintended hash collisions. In fact this idea of bit suppression is the key to producing more secure pseudonyms. The details are presented in the following section.

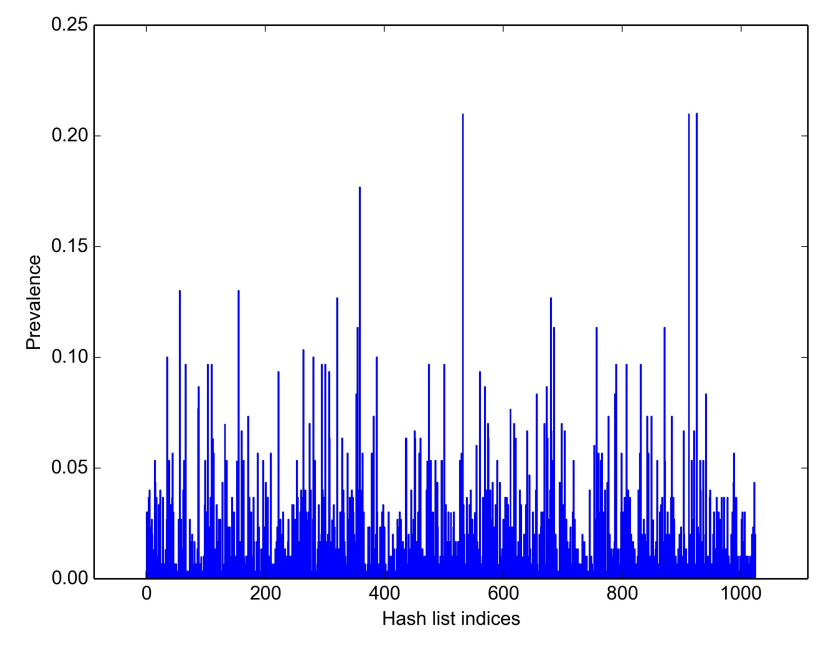

Figure 3. Proportion of occurrences of smallest hash values for sample data

\subsection{1-bit hashing}

An alternative to returning the full hash value for minwise hashing is to return a fixed number of bits, say the $b$ least significant bits. Li and König [13] use $b$-bit hashing to reduce storage costs, their application being the hashing of large text corpora. For our purposes $b$-bit hashing can be used both to disguise the sizes of token sets, and to mitigate against frequency attacks on the individual hash functions. By restricting the number of published bits we purposefully generate hash collisions that do not correspond to matching tokens.

The additional collisions require a new estimator for the Jaccard score. A rather complex estimator is presented in Li and König's paper. But here we consider only 1-bit hashing where the chosen bit is the least significant bit, and provide a justification for a simpler mathematical treatment. We follow the implementation detailed earlier, but simply discard all but the least significant bit from each of the minwise hashes. Thus the 1-bit hash for a hash function $h$ (from a minwise independent family) and token set $S$ is, 


$$
H(S)=\min _{x \in S}\{h(x)\} \& 1
$$

The 1-bit hashes for a sequence of hash functions are concatenated to produce a single $m$-bit hash. This hash has highly desirable properties and admits a simple estimator and a simple closed form expression for its variance. We term this a 'concatenated 1-bit hash' to distinguish it from a 1-bit hash associated with a single hash function.

\subsubsection{Marginal probability of a zero bit}

The first issue to address is the probability of generating a 0 as a 1-bit hash from a token set of size $n$ and randomly generated 1-bit minwise hash function $H$. This probability is trivially 0.5 for $n=1$, so the remainder of this section considers the case of $n>1$. In this section we show that the probability can be taken as 0.5 for all practical values of $q$ and $n$. In Section 3.4.2 we use this result to derive a simple estimator for the Jaccard score and a closed form expression for its variance.

The minwise hash value is a first order statistic which is distributed,

$$
P\left(X_{(1)}=x\right)=\left(1-\frac{x}{2^{q}}\right)^{n}-\left(1-\frac{x+1}{2^{q}}\right)^{n} .
$$

If we take the least significant bit of a minwise hash as our 1-bit hash, then the probability that the 1-bit hash is 0 is equal to the following summation,

$$
P(H(S)=0)=\sum_{x=0,2, \ldots}^{2^{q}-2}\left[\left(1-\frac{x}{2^{q}}\right)^{n}-\left(1-\frac{x+1}{2^{q}}\right)^{n}\right]
$$

and the probability that the 1-bit hash is 1 is the following,

$$
P(H(S)=1)=\sum_{x=1,3, \ldots}^{2^{q}-1}\left[\left(1-\frac{x}{2^{q}}\right)^{n}-\left(1-\frac{x+1}{2^{q}}\right)^{n}\right]
$$

or

$$
P(H(S)=1)=\sum_{x=0,2, \ldots}^{2^{q}-2}\left[\left(1-\frac{x+1}{2^{q}}\right)^{n}-\left(1-\frac{x+2}{2^{q}}\right)^{n}\right] .
$$

Let $f(x)=\left(1-\frac{x}{2^{q}}\right)$. It is simple to show that $f(x)$ is both monotonically decreasing and convex on the interval $\left[0,2^{q}\right]$. Thus,

$$
f(x)-f(x+c)>f(y)-f(y+c)
$$

for all $0 \leq x<y$ and constant $c$ s.t. $y+c \leq 2^{q}$. Therefore, for finite $q$ and $n>1$,

$$
P(H(S)=0)>P(H(S)=1) .
$$

Furthermore, the summations can be rearranged, 


$$
\begin{gathered}
P(H(S)=0)=f(0)-f(1)+\sum_{x=2,4, \ldots}^{2^{q}-2}[f(x)-f(x+1)] \\
P(H(S)=0)=f(0)-f(1)+\sum_{x=0,2, \ldots}^{2^{q}-4}[f(x+2)-f(x+3)] \\
P(H(S)=1)=f\left(2^{q}-1\right)-f\left(2^{q}\right)+\sum_{x=0,2, \ldots}^{2^{q}-4}[f(x+1)-f(x+2)] .
\end{gathered}
$$

The aforementioned properties of $f(x)$ imply that,

$$
\sum_{x=0,2, \ldots}^{2^{q}-4}[f(x+1)-f(x+2)]>\sum_{x=0,2, \ldots}^{2^{q}-4}[f(x+2)-f(x+3)]
$$

thus,

$$
P(H(S)=1)-f\left(2^{q}-1\right)+f\left(2^{q}\right)>P(H(S)=0)-f(0)+f(1)
$$

and as $P(H(S)=0)+P(H(S)=1)=1$ we have the following upper bound on $P(H(S)=0)$,

$$
U=1-\frac{1}{2}\left[\left(\frac{1}{2^{q}}\right)^{n}+\left(1-\frac{1}{2^{q}}\right)^{n}\right]>P(H(S)=0) .
$$

$U$ is by no means the tightest upper bound on $P(H(S)=0)$ that can be derived. Properties of $f^{\prime}(x)$ can be exploited to achieve tighter bounds. But the easily derived upper bound above allows examination of the asymptotics of $P(H(S)=0)$.

It is clear that for any fixed $n$ as $q \rightarrow \infty$ then $P(H(S)=0) \rightarrow \frac{1}{2}$; and for any fixed $q$ as $n \rightarrow \infty$ then $P(H(S)=$ $0) \rightarrow 1$. We can use Bernoulli's inequality,

$(1+x)^{r}>1+r x$ for integer $r \geq 2$ and real $x \geq-1, x \neq 0$

to generate a looser bound,

$$
1-\frac{1}{2^{q n+1}}-\frac{1}{2}\left(1-\frac{n}{2^{q}}\right)>P(H(S)=0)
$$

This bound makes it clear that for $n \ll 2^{q}$ and large enough $q n, P(H(S)=0)$ will be very close to 0.5 . A high precision floating point library was used to generate $U$ to 200 bits of precision for $q=64$ and various values of $n$. When the results were truncated to 53 bits of precision (i.e. the precison of a standard 64-bit float) they were equal to 0.5 for all $n<=2048$. Even for $q=64$ and $n=1000000 U$ was only 0.5000000000000271 . In practice the assumption that $P(H(S)=0)=P(H(S)=1)$ is reasonable for $q=64$ and any plausible token set size. This also demonstrates that concatenated 1-bit hashes can be treated as uniform random integers for reasonable values of $q$.

It should be emphasised that these results hold for the 1-bit hash produced by taking the least significant bit. In principle other bit positions could be selected, but $q$ would need to be larger in order for the above assumption to be reasonable. 


\subsubsection{Jaccard score estimation}

Assume that the probability of an unintended hash collision on the full $q$-bit hash is negligible. Then the full minwise hashes for a pair of token sets are equal with probability equal to the Jaccard score. If we also assume that the marginal probability of a 0 for a 1-bit hash is 0.5 , then the probability of a collision on the least significant bits is,

$$
P\left(H\left(S_{A}\right)=H\left(S_{B}\right) \mid q\right)=J_{A, B}+\left(1-J_{A, B}\right)\left(\frac{1}{2}-\frac{1}{2^{q}}\right)
$$

For sufficiently large $q, 1 / 2^{q}$ is negligible and,

$$
P\left(H\left(S_{A}\right)=H\left(S_{B}\right)\right)=J_{A, B}+\frac{1}{2}\left(1-J_{A, B}\right)
$$

leading to the estimator,

$$
\widehat{J}_{A, B}=2 \frac{X}{m}-1
$$

where $X$ is the number of collisions on the least significant bit.

We can also express this in terms of the hamming distance of a pair of concatenated 1-bit hashes,

$$
\widehat{J}_{A, B}=1-\frac{2 h_{A, B}}{m}
$$

where the Hamming distance $h_{A, B}$ can be calculated very efficiently using the method due to Wegner [16].

The estimator is a linear function of a Binomial proportion leading to the variance,

$$
\operatorname{Var}\left(\widehat{J}_{A, B}\right)=\frac{\left(1+J_{A, B}\right)\left(1-J_{A, B}\right)}{m}
$$

or

$$
\operatorname{Var}\left(\widehat{J}_{A, B}\right)=\frac{1-J_{A, B}^{2}}{m}
$$

Despite discarding all but the least significant bit in the full hashes, the impact on the variance is relatively minor. 1-bit hashing inflates the variance by a factor (assuming no unintended collisions on the $q$-bit hash),

$$
\frac{\operatorname{Var}_{1}\left(\widehat{J}_{A, B}\right)}{\operatorname{Var}_{q}\left(\widehat{J}_{A, B}\right)}=1+\frac{1}{J_{A, B}}
$$

which tends to 2 as $J_{A, B}$ tends to 1 , but is only 3 for a Jaccard score as low as 0.5 . Note: in record linkage we are generally less interested in precise estimation for lower scores.

This reflects the main finding in Li and König [13]. They showed that Jaccard score estimates at a given level of precision could be generated using less storage if they generated more minwise hashes and discarded all but a subset of bits. 
Table 2. 1-bit hashes corresponding to the minwise hashes in Table 1

\begin{tabular}{|c|ccccccc|}
\hline & $\mathrm{H}_{1}$ & $\mathrm{H}_{2}$ & $\mathrm{H}_{3}$ & $\mathrm{H}_{4}$ & $\mathrm{H}_{5}$ & $\ldots$ & $\mathrm{H}_{\mathrm{m}}$ \\
\hline $\mathrm{S}_{1}$ & 1 & 0 & 1 & 0 & 0 & & \\
$\mathrm{~S}_{2}$ & 1 & 1 & 1 & 0 & 0 & & \\
$\ldots$ & & & & & & & \\
$\mathrm{S}_{\mathrm{N}}$ & & & & & & & \\
\hline
\end{tabular}

\subsubsection{Properties of concatenated 1-bit hashes}

The most important property has been established. For any reasonable value of $q$ a concatenated 1-bit hash can be treated as a $\left[0,2^{m}-1\right]$ uniform random integer. This holds independently of the value of the underlying token set. It follows that:

- The sizes of the underlying token sets cannot be estimated from pseudonyms

- Pseudonyms can be compressed by simply discarding bits in fixed, but arbitrarily chosen bit positions

We have shown that:

- Estimation bias is negligible

- We have a closed form expression for the variance of $\widehat{J}_{A, B}$

As the Jaccard score estimator is a linear function of a Binomial proportion:

- We can easily generate confidence intervals for $J_{A, B}$

- We can specify $m$ to provide a given precision for Jaccard score estimation

Other properties can be illustrated by considering the table of 1-bit hashes in Table 2. This contains the 1-bit hashes corresponding to the minwise hashes in Table 1; each row represents the bits of the corresponding concatenated 1-bit hash.

It is evident that 1-bit hashing generates many additional collisions. Each column in Table 2 can contain at most 2 distinct values. Also, it follows that the expected number of $0 \mathrm{~s}$ in a column is half the number of rows. This does a lot to obfuscate token frequencies and guard against variable re-identification (through the distribution of estimated token set sizes) and frequency attacks on tokens. The attacks illustrated in Section 3.3 are infeasible. Inferences are far weaker than those that could be made with Bloom filters.

Of course, attacks on the complete set of concatenated 1-bit hashes are still possible. As we argued earlier, the risk of frequency attacks on the full set of pseudonyms cannot reasonably be limited via the pseudonymisation scheme itself. This source of risk must be addressed by alternative means which are outside the scope of this paper. 


\subsubsection{XOR compression}

$\mathrm{Li}$ and König [13] provide an estimator for $b$-bit hashes that are compressed via splitting pseudonyms into equal sized chunks and XORing. It is not immediately obvious that this form of compression will provide additional security. However, it might be useful for reducing the size of pseudonyms and reducing storage costs.

With XOR compression each published bit is generated by a pair of 1-bit minwise hash functions. For example, take the bitstring (concatenated 1-bit hash),

\section{1}

split into two equal sized chunks and XOR.

$$
1011 \oplus 0101=1110
$$

Let $P\left(H_{i}\left(S_{A}\right)=H_{i}\left(S_{B}\right)\right)$ be denoted $P$ and the probability of a collision on a pair of XORed 1-bit minwise hashes (for any given bit position) be denoted $P_{2}$. Assume we have two pairs of bits $(a, b),(c, d)$ where $P(a=c)=P(b=$ $d)=P$. Then the probability $P_{2}$ that $P(a \oplus b)=P(c \oplus d)$ is,

$$
P_{2}=P^{2}+(1-P)^{2} .
$$

That is, $a \oplus b$ will collide with $c \oplus d$ if $a=c$ and $b=d$, or if $a \neq c$ and $b \neq d$.

$\mathrm{Li}$ and König solve this quadratic and produce an estimator for the Jaccard score. The relevant root can be identified by noting that $0 \leq J_{A, B} \leq 1$, and so $P \geq 1 / 2$,

$$
P=\frac{1+\sqrt{2 P_{2}-1}}{2}
$$

which can be more usefully rearranged,

$$
2 P-1=\left(2 P_{2}-1\right)^{\frac{1}{2}} .
$$

Let $X_{2}$ denote the number of collisions between a pair of XOR compressed 1-bit minwise hashes of length $m_{2}=$ $m / 2$. Then,

$$
\widehat{J}_{A, B}=\left(2 \frac{X_{2}}{m_{2}}-1\right)^{\frac{1}{2}} .
$$

This is equivalent to the estimator provided by $\mathrm{Li}$ and König - given the simplifying assumptions we justified earlier. However, it is possible to generalize this result to any positive integer $N$.

Assume we have two sequences of bits of length $N$ that are XORed to produce corresponding bits in a pair of compressed 1-bit minwise hashes. The bits in the compressed hashes will collide if, and only if, the number $x$ of non-colliding bits in the original sequences is even. Thus the probability $P_{N}$ of a collision on the compressed bits is the sum of the relevant terms in the Binomial expansion for $(P+(1-P))^{N}$,

$$
P_{N}=\sum_{x=0,2, \ldots}^{N}\left(\begin{array}{l}
N \\
x
\end{array}\right) P^{N-x}(1-P)^{x} .
$$


We note that the terms in the Binomial expansion for $(P-(1-P))^{N}$ are the same as those for $(P+(1-P))^{N}$ except for alternating signs. This leads directly to,

$$
\begin{gathered}
P_{N}=\frac{(P+(1-P))^{N}+(P-(1-P))^{N}}{2} \\
2 P-1=\left(2 P_{N}-1\right)^{\frac{1}{N}}
\end{gathered}
$$

Thus for a positive integer $N$ we have the estimator,

$$
\widehat{J}_{A, B}=\left(2 \frac{X_{N}}{m_{N}}-1\right)^{\frac{1}{N}}
$$

We can use the Taylor expansion approach to generate an approximate expression for the variance of this estimator,

$$
\operatorname{Var}\left(\widehat{J}_{A, B}\right) \approx \frac{1}{N^{2} m_{N}}\left(\frac{1}{J_{A, B}^{2 N-2}}-J_{A, B}^{2}\right) .
$$

Again, this is consistent with the variance derived by $\mathrm{Li}$ and König for $N=2$ given our simplifying assumptions. For the expectation we can again use the Taylor expansion approach,

$$
E\left(\widehat{J}_{A, B}\right) \approx J_{A, B}-\frac{N-1}{N^{2}} \frac{1}{2 m_{N}}\left(\frac{1}{J_{A, B}^{2 N-1}}-J_{A, B}\right) .
$$

$\mathrm{Li}$ and König provide no expression for the expectation of their estimator for $N=2$, but note the bias induced by its non-linearity. The relevance for $\mathrm{Li}$ and König's application is that compressed hashes provide lower variance estimates (for a published pseudonym of given length) for Jaccard scores above a threshold. For $N=2$ this threshold is approximately 0.5774 .

Figures 4 and 5 show the behaviour of the variance and expectation of estimates based on the above approximate expressions. The approximations can be inaccurate for small $m_{N}$ (less than about 1024), and for smaller Jaccard scores, but they can be used to illustrate the impacts of various choices for $N$.

As $J_{A, B} \rightarrow 1$ the variance reduction factor tends to $N$, but variances are significantly inflated for low Jaccard scores. Bias is negligible for larger Jaccard scores, but significant for lower scores. For lower scores we have a non-negligible chance of proportions of collisions less than 0.5. In these cases the estimate is taken as 0 . This truncation reduces both the variance and the bias.

For record linkage we might have situations where XOR compression is warranted. Record linkage can be improved by allocating low probability matches to a set of incorrect matches before using more advanced linkage approaches on the remaining record pairs. Record pairs might be classified as either incorrect or possible matches on the basis of a relatively high similarity score threshold. Shorter pseudonyms - that allow higher scores to be estimated with an adequate degree of precision - will generally make the process more efficient. There might be some use cases where storage costs are also relevant.

As far as security is concerned there appears to be little obvious incentive for using XOR compressed pseudonyms. XOR compressed concatenated 1-bit hashes have similar properties to uncompressed hashes. Considered in isolation a compressed hash is still a random integer. It is notable that XOR compression does not result in the compressed pseudonym filling with 1s, unlike the OR compression used in chemical fingerprinting folding [10]. 


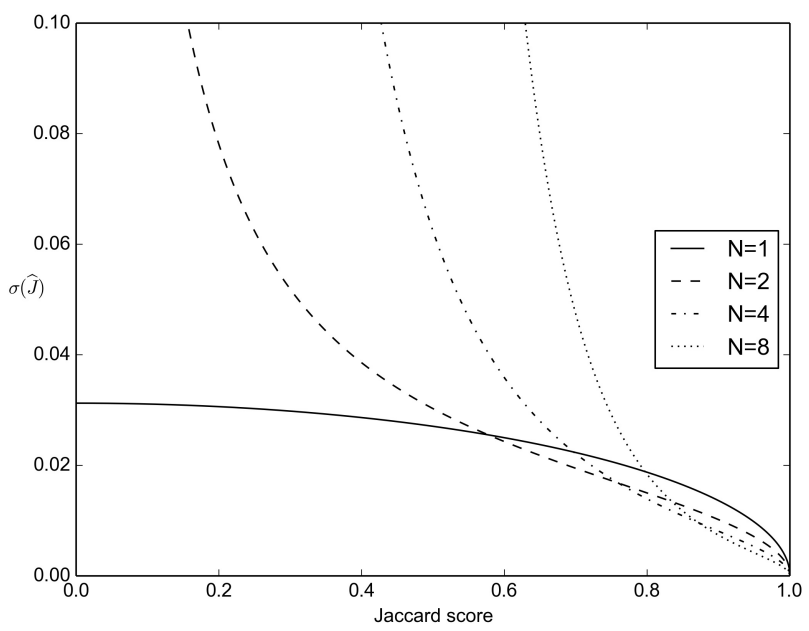

Figure 4. Standard error of estimated Jaccard scores against true scores for varying levels of compression and $\mathrm{m}_{\mathrm{N}}=1024$

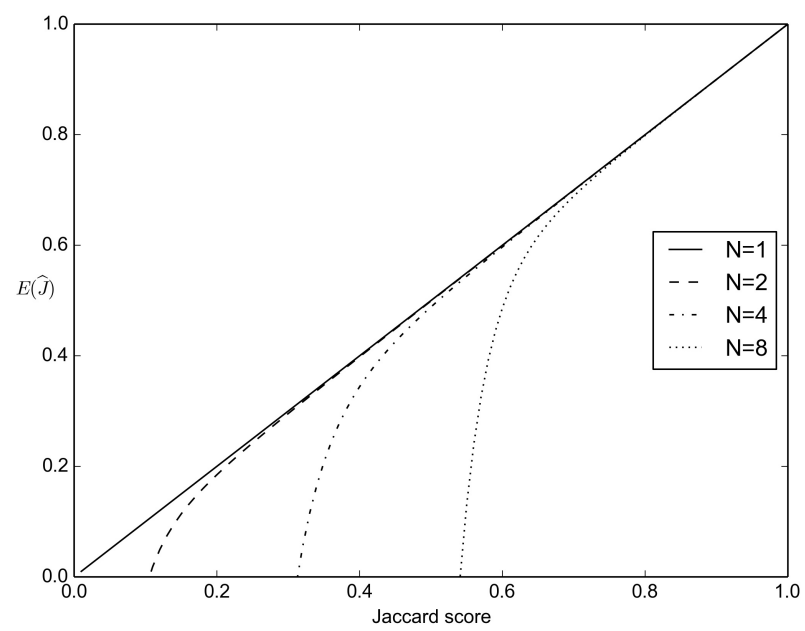

Figure 5. Expected value of estimated Jaccard scores against true scores for varying levels of compression and $\mathrm{m}_{\mathrm{N}}=1024$ 


\section{A comparison of approaches}

The approaches described in this paper were compared using a sample of (not necessarily distinct) 150 Titanic passenger surnames. A second set of surnames was produced by perturbing these names to simulate typographical errors using the following scheme:

1. Transpose 2 adjacent characters with probability $1 / 20$.

2. Delete a character with probability $1 / 60$.

3. Double up a character with probability $1 / 60$.

4. Substitute a character with a keyboard adjacent character with probability $1 / 60$.

5. Delete a character with probability $1 / 60$.

Perturbations were applied in the order given above. Characters (for e.g. deletion) or character pairs (for e.g. transposition) were selected with equal probability.

All strings had leading and trailing underscores added before tokenising into bigrams. Each exact string was compared with each perturbed string using the Bloom filter, minwise hashing and 1-bit hashing approaches. Estimated Jaccard scores were plotted against the exact Jaccard scores generated from the underlying token sets. The results are shown in Figure 6.
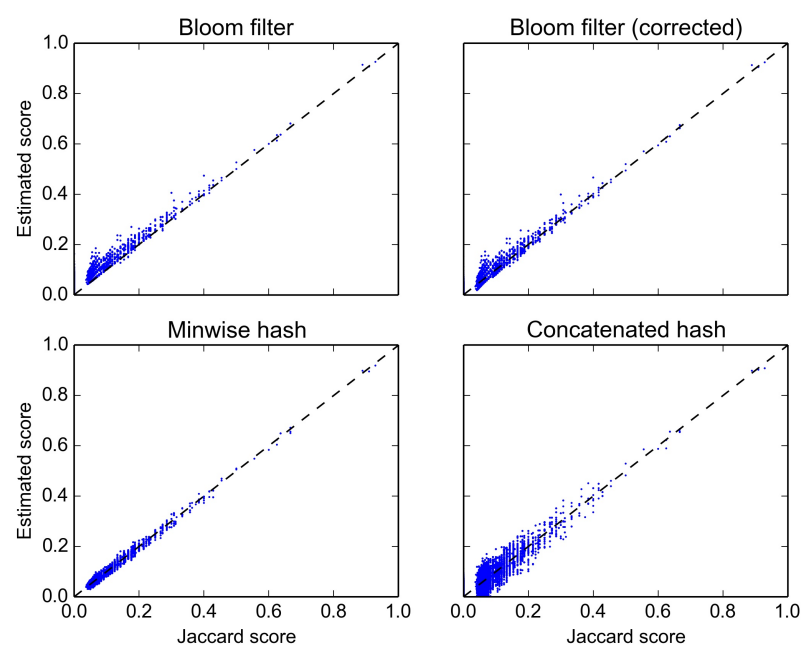

Figure 6. Estimated Jaccard scores against true Jaccard scores for alternative pseudonymisation schemes

A 1024-bit Bloom filter was used with 4 hash functions and the double hashing scheme due to Kirsch and Mitzenmacher [17]. Estimates from the Bloom filter were also generated using the corrected estimator due to Swamidass and Baldi [10]. Minwise hashing used the previously described scheme with 1024 hash functions.

The biasedness of the (uncorrected) Bloom filter estimates is apparent. The minwise hash has smallest variance (at least for lower Jaccard scores), but requires 64 times as much storage as any of the other approaches. The concatenated 1-bit hash estimates have largest variance, but are similar to the (uncorrected) Bloom filter estimates in terms of mean square error (due to the negligible bias).

It should be noted that the (uncorrected) Bloom filter estimates can exhibit large bias and large variance for poor choices of $k$. The value used above was derived via some experimentation. This showed that values designed to minimise the false positive rate (with respect to its use as a set data structure) tended to be very poor for Jaccard score estimation. It is also worth noting that the bias corrected estimator was far less sensitive to choice of $k$. 
An empirical evaluation of XOR compression was performed using a database containing 6100 English surnames ${ }^{4}$. These were perturbed as detailed above, except that each perturbation probability was multiplied by 4 . As we are mainly interested in the behaviour for larger Jaccard scores the comparisons were made only between each surname and its perturbed twin. Comparisons were made between a Bloom filter and XOR compression for $N$ equal to 1 (no compression), 2 and 4. For the Bloom filter we used $k=4$ and the double hashing scheme due to Kirsch and Mitzenmacher [17]. Estimation for the Bloom filter used the bias corrected estimator due to Swamidass and Baldi [10].
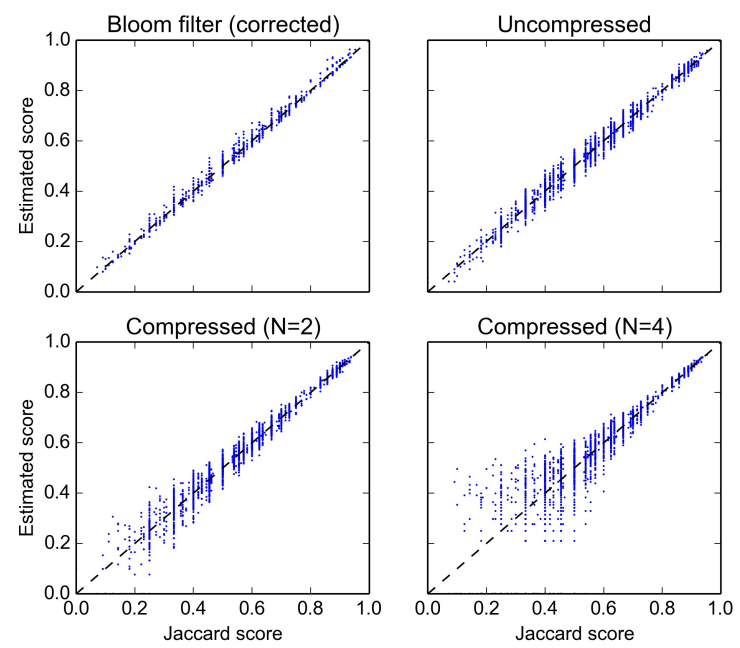

Figure 7. Comparison of Bloom filter and concatenated 1-bit hashes with various degrees of compression

2290 of the surnames were subjected to some degree of perturbation. For the concatenated 1-bit hashes we see the expected relationship with degrees of XOR compression. For $N=4$ the variance is similar to that for the Bloom filter for scores above around 0.8. For lower scores the variance is significantly inflated. Truncation of estimates does a lot to reduce both bias and variance. The numbers of truncated estimates are 1,18 and 226 for $N$ equal to 1 (uncompressed), 2 and 4 respectively. The results are broadly consistent with the plots in Figures 4 and 5.

\section{Discussion}

Privacy-preserving record linkage requires the pseudonymisation of strings before they are used for linkage. Pseudonyms that can convey similarity information allow better record linkage performance. Existing pseudonymisation schemes are vulnerable to attacks that can allow the identification of both variables and tokens.

Bloom filters allow token set lengths to be estimated, and the distribution of token lengths can help to identify the relevant variable. The same is true of pseudonyms consisting of lists of minwise hashes. Bloom filters and lists of minwise hashes allow common tokens to be identified via commonly set bit positions and common hashes respectively. The most obvious benefit offered by lists of minwise hashes is negligible bias in the estimation of Jaccard scores. Even this apparent advantage is a little questionable as it has only been demonstrated to be of practical significance for chemical fingerprinting folding [10]. In any case, the estimation bias for Bloom filters can be very much reduced by using a corrected estimator. This also makes the Bloom filter approach much less sensitive to choice of $k$.

The real advantages of minwise hashing stem from ignoring all but the least significant bit and concatenating the resulting bits into a single bitstring. It becomes infeasible to estimate the lengths of token sets and much more

${ }^{4}$ http: //scrapmaker.com/download/data/wordlists/names/surnames . txt (accessed 14th June 2016) 
difficult to identify tokens through common hashes (as all hashes become either 0 or 1). Jaccard score estimates have negligible bias and known variance. It is also possible to XOR compress concatenated 1-bit hashes to provide lower variance estimates for higher Jaccard scores (for pseudonyms of a given length).

There is an undoubted trade off between the cost of generating pseudonyms and security. For a given precision of estimation Bloom filters are generally shorter than concatenated 1-bit hashes. They also require less hashing to generate. However, in many cases the increased security offered by concatenated 1-bit hashes will far outweigh increased computational cost and space requirements. The use of concatenated 1-bit hashes might also reduce, or eliminate, the need for more complex protocols or further steps to protect the Bloom filters. Protecting Bloom filters using, for example, homomorphic encryption [18] can also increase computational demands.

One issue that has not been discussed is the search for pseudonyms that are sufficiently similar to a target pseudonym. In record linkage the number of record pairs can be very large, and it useful to be able to restrict the full record linkage machinery to only those pairs that are sufficiently similar. Techniques using binary trees have been developed within the chemical fingerprinting literature [19] and applied to record linkage [20]. Essentially, Bloom filters can be added to a binary tree where left/right children correspond to 0/1 bits. Each distinct path from the source node to a sink node represents a distinct pseudonym. Search starts at the source node and bounds are placed on the Jaccard scores (when compared with the target) as search progresses. Search can be halted at nodes where upper bounds are lower than a predefined threshold. Concatenated 1-bit hashes lend themselves to similar treatment, and there are both advantages and disadvantages compared to Bloom filters. This will be the subject of a future publication.

Another aspect that has only been briefly mentioned is the protocol. It has simply been assumed that Alice and Bob can independently pseudonymise their strings and that matching is subsequently performed by some third party, Carol. Detailed discussion of protocols is outside the scope of this paper. But most protocols that uses Bloom filters can be made more secure by simply substituting concatenated 1-bit hashes. The exceptions would be protocols that are designed to incorporate further protections for the Bloom filters.

In summary, a more secure alternative to Bloom filters has been presented. It is largely based on existing methods that have been used in other application areas. The security benefits have been demonstrated through both theory and example. A simpler mathematical treatment has been justified and this has allowed some existing work (XOR compression) to be generalised. The methodology has been considered in relation to the motivating application area of record linkage. However, it may prove to be useful in other areas such as chemical fingerprinting. An open source Python implementation is available at https://github.com/DuncanSmith147/pseudonymization.

\section{References}

[1] I. Fellegi, A. Sunter, A theory for record linkage, JASA 64 (238) (1969) 1183-1210.

[2] M. A. Jaro, Advances in record linkage methodology as applied to the 1985 census of Tampa Florida, JASA 84 (406) (1989) $414-420$.

[3] W. E. Winkler, String comparator metrics and enhanced decision rules in the Fellegi-Sunter model of record linkage, in: Proceedings of the Section on Survey Research Methods (American Statistical Association), 1990, pp. 354-359.

[4] W. E. Yancey, Improving EM algorithm estimates for record linkage parameters, in: Proceedings of the Section on Survey Research Methods (American Statistical Association), pp. 3835-3840.

[5] D. Smith, N. Shlomo, Report for the data without boundaries project, Tech. rep., University of Manchester, http://hummedia. manchester.ac.uk/institutes/cmist/archive-publications/reports/2014-01-Data_without_Boundaries_Report.pdf.

[6] P. Contiero, A. Tittarelli, G. Tagliabue, A. Maghini, S. Fabiano, P. Crosignani, R. Tessandori, The EpiLink record linkage software : Presentation and results of linkage test on cancer registry files, Methods Inf Med (44) (2005) 66-71.

[7] M. A. Jaro, Unimatch: A record linkage system: User's manual, Tech. rep., U.S. Bureau of the Census (1976).

[8] T. Churches, P. Christen, Some methods for blindfolded record linkage, BMC Medical Informatics and Decision Making 4 (9).

[9] R. Schnell, T. Bachteler, J. Reiher, Privacy-preserving record linkage using Bloom filters, BMC Medical Informatics and Decision Making 9 (41).

[10] S. Swamidass, P. Baldi, Mathematical correction for fingerprint similarity measures to improve chemical retrieval, Journal of chemical information and modeling 47 (3) (2007) 952-964.

[11] A. Broder, On the resemblance and containment of documents, in: Proceedings of the Compression and Complexity of Sequences, Salerno, Italy, June 11-13, 1997, pp. 21-29.

[12] M. Pătraşcu, M. Thorup, The power of simple tabulation hashing, in: Proceedings of the 43rd annual ACM symposium on Theory of computing, 2011, pp. 1-10.

[13] P. Li, A. König, Theory and applications of b-bit minwise hashing, Communications of the ACM 54 (8) (2011) $101-109$.

[14] M. Wegman, L. Carter, New classes and applications of hash functions, Journal of Computer and System Sciences 22 (3) (1981) $265-279$.

[15] S. Dahlgaard, M. Thorup, Approximately minwise independence with twisted tabulation, in: Proceedings of the 14th Scandinavian Workshop on Algorithm Theory (SWAT), LNCS, Vol. 8503, Springer, Switzerland, 2014, pp. 134-145.

[16] P. Wegner, A technique for counting ones in a binary computer, Communications of the ACM 3 (5) (1960) 322. 
[17] A. Kirsch, M. Mitzenmacherr, Less hashing same performance: Building a better bloom filter, in: Algorithms-ESA 2006, LNCS, Vol. 4168, Springer, Heidelberg, 2006, pp. 456-467.

[18] S. M. Randall, A. P. Brown, A. M. Ferrante, J. H. Boyd, J. B. Semmens, Privacy preserving record linkage using homomorphic encryption, in: First International Workshop on Population Informatics for Big Data (PopInfo. 15), Aug 10, Sydney, 2015.

[19] T. Kristensen, J. Nielsen, C. Pedersen, A tree-based method for the rapid screening of chemical fingerprints, Algorithms for Molecular Biology 5 (9).

[20] T. Bachteler, J. Reiher, R. Schnell, Similarity filtering with multibit trees for record linkage, Tech. rep., German Record Linkage Center, working Paper Series No. WP-GRLC-2013-01. 